\title{
Bifurcation and chaos of a density dependent Leslie population model
}

\author{
Guo Feng $^{1}$ and Song Xinghao ${ }^{1}$
}

${ }^{1}$ Affiliation not available

May 5, 2020

\begin{abstract}
A discrete-time Leslie model with Hassell growth function for two generations is investigated by qualitative analysis and numerical simulation. Local stability analysis of the system is carried out. Many forms of complex dynamics are observed, including chaotic bands with periodic windows, flip bifurcations, and Hopf bifurcations, attractor crises, and non-unique dynamics (meaning that several attractors coexist). Numerical simulation results not only show the consistence with the theoretical analysis but also display the new and interesting dynamical behaviors, including different periodic orbits in chaotic regions, attracting invariant circle, period-doubling bifurcations from stable equilibriums leading to chaos, interior crisis and boundary crisis. The largest Lyapunov exponents are numerically computed to confirm further the complexity of these dynamic behaviors. The analysis and results in this paper are interesting in mathematics and biology.
\end{abstract}

\section{Hosted file}

Bifurcation and chaos of a density dependent .pdf available at https://authorea.com/users/ 303021/articles/434358-bifurcation-and-chaos-of-a-density-dependent-leslie-populationmodel 